\title{
Von der Betten- zur
}

\section{Preissteuerung}

\section{Die Steuerung von Krankenhausleistungen nach dem Ende der dualen Finanzierung}

\section{WULF-DIETRICH LEBER, DAVID SCHELLER-KREINSEN}

Dr. Wulf-Dietrich Leber leitet die Abteilung Krankenhäuser beim GKV-Spitzenverband, Berlin.

Dr. David Scheller-Kreinsen ist Referent Krankenhausvergütung in der Abteilung Krankenhäuser beim GKVSpitzenverband, Berlin.

Das Ende der dualen Finanzierung ist nahe. Setzt sich der Trend rückläufiger Länderausgaben für Krankenhausinvestitionen fort, so wird in rund zehn Jahren die Nulllinie erreicht. Schon heute wird entgegen der gesetzlichen Regelung - rund die Hälfte der Krankenhausinvestitionen via DRG-Vergütung durch die Krankenkassen bezahlt. Eine Diskussion über die Steuerung stationärer Leistungen und die künftige Form der Krankenhausplanung ist überfällig. Traditionelle Planung wird es wahrscheinlich nur noch für Notfallleistungen geben. Für elektive Leistungen müssen neue Steuerungsoptionen diskutiert werden, bei denen angesichts der schwindenden Steuerungswirkung traditioneller Bettenplanung vermehrt Preismechanismen und Abrechnungslizenzen von Bedeutung sein werden.

\section{Erosion der Investitionskostenfinanzierung}

Die Investitionskosten von Krankenhäusern werden nur noch in einem sehr geringen Umfang durch die Länder abgedeckt (Leber, Wolff 2012). So ist der Finanzierungsanteil der Krankenhausausgaben durch die Bundesländer seit der Einführung im Krankenhausfinanzierungsgesetz (KHG) 1972 von über 20 Prozent auf unter fünf Prozent in 2009 geschrumpft. De facto liegt der Wert sogar unter vier Prozent, da in den neuen Bundesländern ein Teil der Länderinvestitionen über Artikel $14 \mathrm{Ge}$ sundheitsstrukturgesetz (GSG) ${ }^{2} \mathrm{GKV}$ finanziert wird. Setzt sich der Rückgang des Finanzierungsanteils der Bundesländer linear fort, wird die duale Finanzierung 2021 die Nulllinie erreichen (siehe Abb. 1).
Aus der 1972 eingeführten dualen Krankenhausfinanzierung wird daher schleichend eine monistische Krankenhausfinanzierung zulasten der Krankenkassen. Dies geschieht allerdings, ohne dass die Krankenkassen vom Gesetzgeber für die Investitionskostenfinanzierung vorgesehen sind, eine entsprechende regelhafte Grundlage definiert wurde oder die Krankenkassen Einfluss auf Standort und Investitionsentscheidungen nehmen können.

1 Gesetz zur wirtschaftlichen Sicherung der Krankenhäuser und zur Regelung der Krankenhauspflegesätze (Krankenhausfinanzierungsgesetz - KHG) vom 29.06.1972, neugefasst durch Bek. v. 10.04.1991(BGBl. I S. 886), zuletzt geändert durch Art. 13 Abs. 1 G v. 12.04.2012 (BGBl. I S. 579).

2 Gesetz zur Sicherung und Strukturverbesserung der gesetzlichen Krankenversicherung (Gesundheitsstrukturgesetz - GSG) vom 21.12.1992 (BGBl. I S. 2266), zuletzt geändert durch Art. 205 V v. 25.11.2003 (BGBl. I S. 2304). 


\section{Abbildung 1: Erosion der dualen Finanzierung 1991 bis 2010, Trendprognose 2021 [1]}

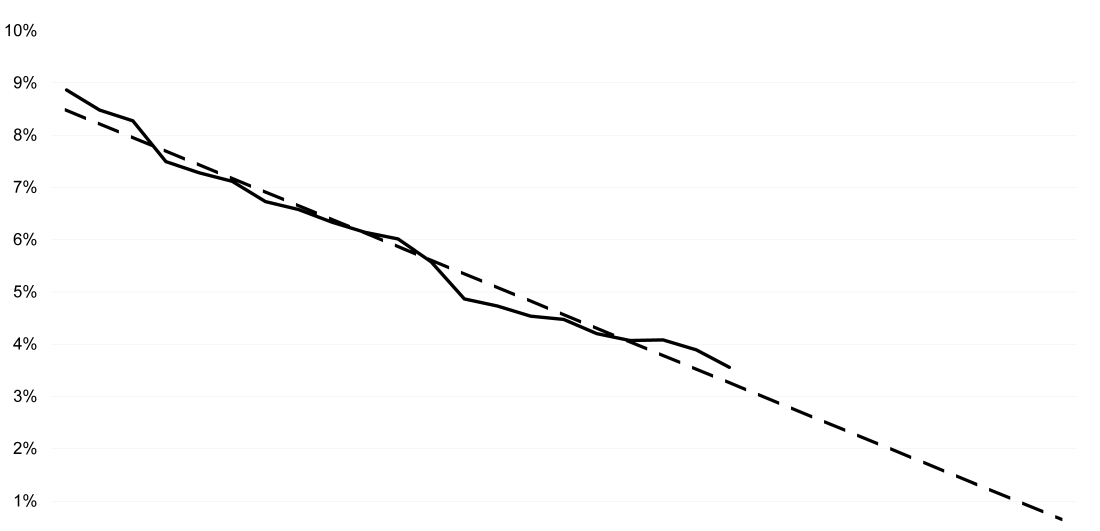

$0 \%$

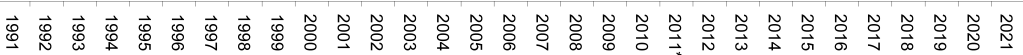

Die mangelnde Investitionsfinanzierung durch die Bundesländer geht einher mit Debatten über einen Investitionsstau in Krankenhäusern. Inzwischen betont auch die Krankenhausseite, dass es sich um einen klaren Fall von Länderversagen handelt. Gleichzeitig ist anzunehmen, dass die mangelnde Investitionskostenfinanzierung aus anderen Quellen kompensiert wird - vornehmlich aus Erlösen der Krankenbehandlung, den Betriebsmitteln. Jedoch stellt die Verwendung von Betriebsmitteln laut KHG für Investitionen eine Zweckentfremdung ohne gesetzliche Grundlage dar, bei der die Krankenkassenaufsicht erlöskürzend einschreiten müsste.

\section{Die mangelnde Investitions- finanzierung durch die Bundes- länder geht einher mit Debatten über einen Investitionsstau in Krankenhäusern.}

\section{Investitionsbewertungs- relationen}

Die Debatte um eine Verbesserung der Pauschalförderung mündete 2009 in eine durch das Krankenhausfinanzierungsreformgesetz (KHRG) $)^{3}$ auf den Weg gebrachte Entwicklung von Investitionsbewertungsrelationen. $\mathrm{Zu}$ künftig sollen pauschale Fördermittel nicht mehr vorrangig nach Betten, sondern leistungsorientiert

Grundsätzlich können die Bundesländer zwei unterschiedliche Mechanismen zur Verteilung der Investitionsmittel nutzen: 1. die krankenhausspezifische Einzelförderung (z. B. der Bau eines OPTrakts) und 2. die Pauschalförderung. Das bei Landes- und Lokalpolitikern beliebtere Instrument ist die Einzelförderung, da diese medienwirksam zur Begünstigung von sichtbaren Projekten, wie z. B. der Erweiterung einer pädiatrischen Einrichtung, eingesetzt werden kann. Gerade deshalb ist die Einzel- kenhaus (InEK) eine detaillierte Grundlage für die Kalkulation erarbeitet, die alle Investitionsmittel unabhängig von ihrer Herkunft berücksichtigt. Rund 30 Häuser beteiligen sich derzeit an der Kalkulation und schon für 2013 könnte dann erstmals ein Set von Investitionsbewertungsrelationen vorliegen.

Generell ist jedoch fraglich, ob diese mit viel Aufwand betriebene und auf hohem technischen Niveau angelegte Reform zu einer Verbesserung der Krankenhausfinanzierung beitragen wird. Denn mehre Länder haben schon mehr oder weniger offiziell erklärt, dass sie diese Investitionsbewertungsrelationen nicht verwenden werden (siehe Tab. 1).

Das Gesetz schreibt die Anwendung von Investitionsbewertungspauschalen jedoch nicht vor, es sorgt lediglich dafür, dass ein entsprechendes technisches Instrument zur Verfügung stehen wird. $\mathrm{Zu}-$ dem bewegen sich die Fördermittel auf einem sehr niedrigen Niveau. Angesichts der mangelhaften Investitionsförderung können daher auch hoch professionell kalkulierte Investitionsbewertungsrelationen nur begrenzten Nutzen stiften. Viele Akteure bezweifeln daher grundsätzlich, dass es sich lohnt, auf diesem Niveau über die Verteilungsmechanismen zu streiten. Darüber hinaus kann die Einzelförderung die Verteilung der Investitionsmittel so stark verzerren, dass es auf die Rationalität der Pauschalförderung auch nicht mehr ankommt.

Es stellt sich daher die Frage, warum die Kalkulation mit derart großem Aufwand betrieben wird. Auf Kassenseite besteht der Verdacht, dass die Kalkulation nur dazu dient, eine geordnete Übergabe der Investitionskostenfinanzierung von den Länderhaushalten auf die Krankenkassen zu übertragen. Pauschale Krankenhausförderung ist politisch wenig ertragreich und belastet lediglich den Landeshaushalt. Dafür spricht auch, dass alles, was das InEK kalkuliert, bislang von den Krankenkassen bezahlt worden ist. Es liegt daher nahe, die Investitionskosten „leistungsorientiert" in die Krankenhausentgelte $\mathrm{zu}$ integrieren.

Wie eingangs dargestellt wurde die Finanzierung der Investitionskosten de facto schon längst auf die Kassen übertragen. Eine offizielle Statistik gibt es

\footnotetext{
Gesetz zum ordnungspolitischen Rahmen der Krankenhausfinanzierung ab dem Jahr 2009 (Krankenhausfinanzierungsreformgesetz - KHRG) vom 17.03.2009 (BGBl. I S. 534).
} 
Tabelle 1: Die Einführung von leistungsorientierten Investitionsbewertungspauschalen in den Bundesländern, Ergebnisse einer Befragung durch f\&w 2012 [2]

\begin{tabular}{|l|c|}
\hline Bundesland & $\begin{array}{c}\text { Einführung von leistungsorientierten Investitions- } \\
\text { bewertungspauschalen }\end{array}$ \\
\hline Baden-Württemberg & nein \\
\hline Bayern & nein \\
\hline Berlin & $\begin{array}{c}\text { Erfahrungen der anderen Bundesländer abwarten, } \\
\text { dann entscheiden }\end{array}$ \\
\hline Brandenburg & ja \\
\hline Bremen & ja \\
\hline Hamburg & nein \\
\hline Hessen & ja \\
\hline Mecklenburg-Vorpommern & nein \\
\hline Niedersachsen & keine Stellungnahme \\
\hline Nordrhein-Westfalen & umgesetzt* \\
\hline Rheinland-Pfalz & nein \\
\hline Saarland & ja \\
\hline Sachsen & nein \\
\hline Sachsen-Anhalt & ja \\
\hline Schleswig-Holstein & nein** \\
\hline Thüringen & nein \\
\hline
\end{tabular}

Legende:

* laut Eigendarstellung

**Antwort vom 30.04.2012 (vor der Landtagswahl)

nicht. Aber wenn man einmal annimmt, dass die wahre Investitionsquote zehn Prozent der Krankenhauskosten beträgt, dann wird derzeit schon mehr als die Hälfte der Investitionen via DRGs finanziert. Das wirft die Frage auf, ob die derzeitigen DRG-Relativgewichte, die ja explizit keine Investitionsaufwände enthalten, den Aufwand richtig abbilden. Wer aufwandsgerecht finanzieren will, der müsste eigentlich schon heute die Hälfte der notwendigen Investitionsmittel in die DRG-Kalkulation einbeziehen. In einer solchen Betrachtungsweise sind die gegenwärtigen DRGs eigentlich verzerrt; sie enthalten eine Unterbewertung kapitalintensiver Leistungen und eine Überbewertung personal- und materialintensiver Leistungen.

Würde diese Verzerrung irgendwann öffentlichkeitswirksam diskutiert, dann wäre die Kalkulation von Investitionsbewertungsrelationen vielleicht doch nicht überflüssig. Sie wäre die Basis, um die DRG-Relativgewichte aufwandsgerecht $\mathrm{zu}$ adjustieren. Aber wo sind die politischen Kräfte für eine solche Adjustierung? Die Deutsche Krankenhausgesellschaft (DKG), der bei jeder Veränderung von Relativgewichten Ärger ins Haus steht, wird sie nicht fordern - es sei denn garniert mit Mehrforderungen an die Krankenkassen. Der vermeintliche Investitionsstau wird wahrscheinlich fröhlich propagandistische Urstände feiern. Die Krankenkassen werden diese Korrektur nicht fordern, wenn nicht endlich mit der quasi offiziellen Übernahme der Finanzierungslast auch eine erweiterte Mitsprache bei der Krankenhausplanung einhergeht. Dies führt zum eigentlichen Problem der Investitionsfinanzierung: zur Unmöglichkeit, künftig stationäre Leistungen mittels klassischer bettenorientierter Krankenhausplanung zu steuern.

\section{Der Krankenhausplan: Verletzung der fiskalischen Äquivalenz}

Im Krankenhausbereich fallen Entscheidungen zur Planung und Finanzierung von Leistungen auf mehreren Ebenen problematisch auseinander. Die Bundesländer entscheiden im Rahmen der Krankenhausplanung über wichtige

Parameter der stationären Versorgung, müssen aber nur Teile der entstehenden Kosten verantworten. In der Finanzwissenschaft wird diese Konstellation als Verletzung der fiskalischen Äquivalenz bezeichnet, da der Kreis der Entscheidungsträger, der Nutznießer, sowie der Steuer- bzw. Beitragszahler nicht zusammenfällt und somit eine wichtige Voraussetzung für eine nutzenstiftende Leistungserbringung von Gütern in korporatistischen oder öffentlichen Sektoren nicht gegeben ist (Olson 1969).

Abweichend vom Prinzip der fiskalischen Äquivalenz wird die Krankenhauspolitik von den Ländern zunehmend im verantwortungsfreien Raum ohne finanzielle Erwägung und Haftung gestaltet. Dies spiegelte sich auf Bundesebene zuletzt u. a. in den Auseinandersetzungen zwischen den Ländern und dem Bundesgesundheitsminister über Aspekte der Krankenhausfinanzierung (u. a. die Höhe der Mehrleistungsabschläge und die Einführung des Kostenorientierungswertes) im Gesetzgebungsverfahren des Psych-Entgeltgesetzes (PsychEntgG) ${ }^{4}$ wider. Noch dramatischer ist jedoch die permanente Verletzung der sog. „regionalen Äquivalenz" auf Landesebene. Hier entscheiden die Landesregierungen im Rahmen der Krankenhausplanung über Standorte und Schwerpunkte der stationären

Die Krankenhauspolitik wird zunehmend von den Ländern im verantwortungsfreien Raum ohne finanzielle Erwägung und Haftung gestaltet.

Versorgung. Die Kosten werden jedoch überwiegend bundesweit auf die GKVGemeinschaft umgelegt, da die Betriebsmittel der Kassen zur Finanzierung der laufenden Kosten und größtenteils auch der Investitionskosten herangezogen

4 Gesetz zur Einführung eines pauschalierenden Entgeltsystems für psychiatrische und psychosomatische Einrichtungen (Psych-Entgeltgesetz - PsychEntgG) vom 14.06.2012. BT-Drs. 17/8986 v. 14.03.2012: http://dipbt.bundestag.de/ dip21/btd/17/089/1708986.pdf, BR-Drs. 349/12 v. 15.06.2012: http://www.bundesrat.de/cln_228/nn_8336/SharedDocs/ Drucksachen/2012/0301-400/349-12,te mplateId=raw, property=publicationFile pdf/349-12.pdf. 
werden (siehe Abschnitt 1). Dies bedeutet grob vereinfacht: die Landesregierungen können landesspezifischen Nutzen stiften, ohne die Kosten zu beachten, da diese größtenteils von bundesweit agierenden Kassen ,vergemeinschaftet" werden. Die desaströsen Folgen einer solchen dauerhaften Verletzung des Prinzips der regionalen Äquivalenz erleben wir gerade im Zuge der Eurokrise. Ähnlich wie bei der Gemeinschaftswährung steigen auch im Fall der Krankenhausplanung die volkswirtschaftlichen Kosten, je länger die Fehlanreize wirken und sich in strukturellen Verkrustungen niederschlagen.

Die klassische Krankenhausplanung ist auch aus einem weiteren Grund nicht mehr funktionsfähig: Ihre zentrale Steuerungsgröße - das Krankenhausbett ist kaum noch geeignet, stationäre Kapazitäten abzubilden. Bei gleicher Bettenzahl können sich völlig unterschiedliche stationäre Leistungsvolumina ergeben. In der Anfangsphase der dualen Finanzierung mag das abteilungsspezifisch konkretisierte Bett noch eine halbwegs brauchbare Steuerungsgröße gewesen sein, heute stellt das Bett kaum noch eine Begrenzung des Leistungsvolumens dar. Einige Bundesländer (z. B. SachsenAnhalt) sind deshalb in ihren Krankenhausplanungen dazu übergegangen, das Bett nur noch nachrichtlich mitzuteilen. Geplant werden lediglich Standorte und Abteilungen. Eine solche „Rahmenplanung" führt dazu, dass letztlich keine Kapazitätsplanung mehr existiert, sondern stationäre Leistungen weitestgehend freigegeben werden.

Es gibt den Versuch, Investitionsvolumina an anderen Größen (z. B. Casemix) $\mathrm{zu}$ orientieren. Der Casemix ist jedoch nirgends Gegenstand der Planung! So zeigt eine kurze Analyse, dass das System der dualen Finanzierung aus mehreren Gründen einer Neukonzeption weichen muss:

- Die Investitionen werden nicht mehr (ausreichend) von den Ländern finanziert.

- Das Krankenhausbett ist keine sinnvolle Plangröße mehr und beschränkt die stationäre Leistungserbringung letztlich nicht mehr.

- Bei bundesweit mischkalkulierten Beitragssätzen und regionalem Nutzen (Verletzung der fiskalischen
Äquivalenz) ist auch die „Restaurierung“ der dualen Finanzierung, also auskömmliche Fördervolumina und Beschränkung des Casemix durch die Bundesländer, keine ordnungspolitische Alternative. Überkapazitäten würden nicht abgebaut.

An die Stelle der klassischen bettenorientierten Kapazitätsplanung müssen deshalb andere Steuerungsmechanismen treten. In Zeiten der leistungsorientierten DRG-Vergütung sollten diese an Preisen (Basisfallwerte) und Mengen (Casemix-Volumen) ansetzen.

\section{Preis- und Mengendynamik}

Die alles entscheidende Stellgröße für die Preisentwicklung im Krankenhausbereich ist der Landesbasisfallwert

\section{An die Stelle der klassischen bettenorientierten Kapazitätsplanung müssen andere Steuerungs- mechanismen treten.}

(LBFW). Er wird zwischen Kassen und Landeskrankenhausgesellschaft jährlich für das Folgejahr verhandelt. Für die Verhandlung sind gemäß \$ 10 KHG die Kosten- und Produktivitätsentwicklung zu berücksichtigen. Zudem gibt es bei der Veränderung gegenüber dem Vorjahr eine „schneidende Obergrenze“.
Dies war traditionell die Grundlohnentwicklung ( $\int 71$ SGB V) und soll seit der Regelung im KHRG die Kostenentwicklung sein. Zur Ermittlung der Kosten entwickelt das Statistische Bundesamt ein krankenhausspezifisches Warenkorbkonzept und veröffentlicht das Ergebnis als sog. „Orientierungswert“ jeweils Mitte des Jahres. Das Bundesministerium für Gesundheit (BMG) soll jährlich einen Anteil am Orientierungswert, den sog. „Veränderungswert“ bestimmen, der die Obergrenze für die Verhandlung der Landesbasisfallwerte darstellt. Das BMG kann zudem entscheiden, wann diese neue Kostenorientierung erstmals angewendet wird. Im Ergebnis hat das dazu geführt, dass es bislang nicht zu einer Anwendung dieser Systematik gekommen ist.

Durch das GKV-Finanzierungsgesetz (GKV-FinG) $)^{5}$ wurde die Grundlohnanbindung zunächst um zwei Jahre verlängert. De facto wurde für 2011 eine Begrenzung des Preisanstiegs im stationären Bereich (LBFW) auf eine um 0,25 Prozentpunkte verminderte Grundlohnrate gesetzlich fixiert. Für 2012 wurde eine Begrenzung entsprechend einer um 0,5 Prozentpunkte verringerten Grundlohnrate festgelegt. Das jüngst verabschiedete PsychEntgG wiederum sieht vor, dass

5 Gesetz zur nachhaltigen und sozial ausgewogenen Finanzierung der Gesetzlichen Krankenversicherung (GKV-Finanzierungsgesetz - GKV-FinG) vom 22.12.2010 (BGBl. I S. 2309).

\section{Abbildung 2: Ausgabenentwicklung stationärer Sektor 2000 bis 2012 [3]}

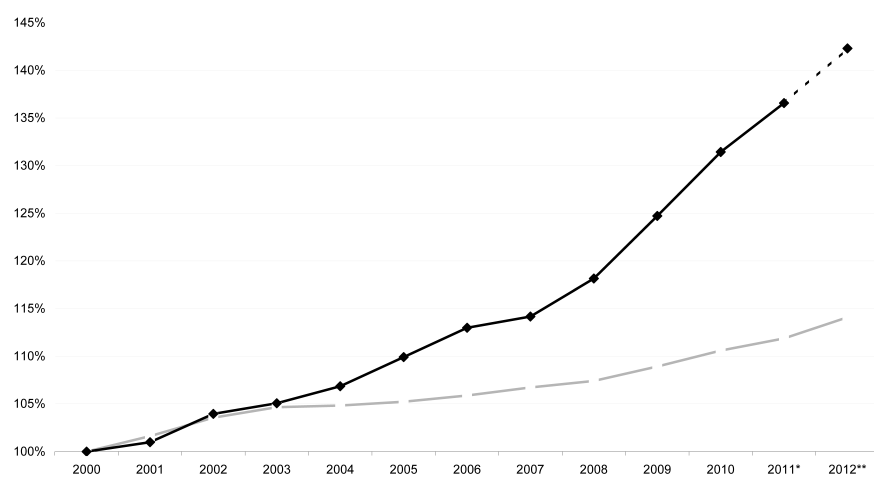

Legende:

Amtliche Statistik KJ1 (2000 - 2010)

*Amtliche Statistik KV45 (2011)

** Schätzungen des GKV-Spitzenverbandes (2012) 
der Orientierungswert wie ursprünglich angedacht vom Statistischen Bundesamt ermittelt und erstmalig zum 30.09.2012 veröffentlicht wird. Die Festlegung des Veränderungswertes soll aber nicht durch den Gesundheitsminister erfolgen, sondern künftig zwischen den Selbstverwaltungspartnern auf Bundesebene bis zum 31.10. des jeweiligen Jahres verhandelt werden.

Für diese Verhandlung ist der gesetzlichen Krankenversicherung, der Privaten Krankenversicherung (PKV) und der Deutschen Krankenhausgesellschaft (DKG) ein Korridor vorgeben, falls der Orientierungswert über der Grundlohnrate liegt: Untergrenze ist in diesem Fall die Grundlohnrate. Die Obergrenze kann hingegen bis zu 33 Prozent der Differenz aus dem vom Statistischen Bundesamt ermittelten Orientierungswert und der Grundlohnrate betragen. Die Festlegung eines Veränderungswertes, der unterhalb der Grundlohnrate liegt, ist lediglich für den unwahrscheinlichen Fall vorgesehen, dass der vollständige Orientierungswert kleiner ist als die Grundlohnrate.

Trotz der bisher geltenden Grundlohnsummenkopplung wurde die Ausgabendynamik in den letzten zehn Jahren kaum gedämpft. Abbildung 2 belegt diese Entwicklung eindrucksvoll. Eine direkte Folge der beschriebenen Preisdynamik war eine substanzielle Mengenausweitung.

Als Konsequenz scheint der Anstieg der stationären Krankenhausfälle in Deutschland inzwischen unumkehrbar. Mit rund 18 Mio. stationären Krankenhausfällen wurde für 2011 ein neuer Höchststand erreicht. Ausgehend von diesem Trend prognostiziert das Statistische Bundesamt bis 2030 (trotz Be-

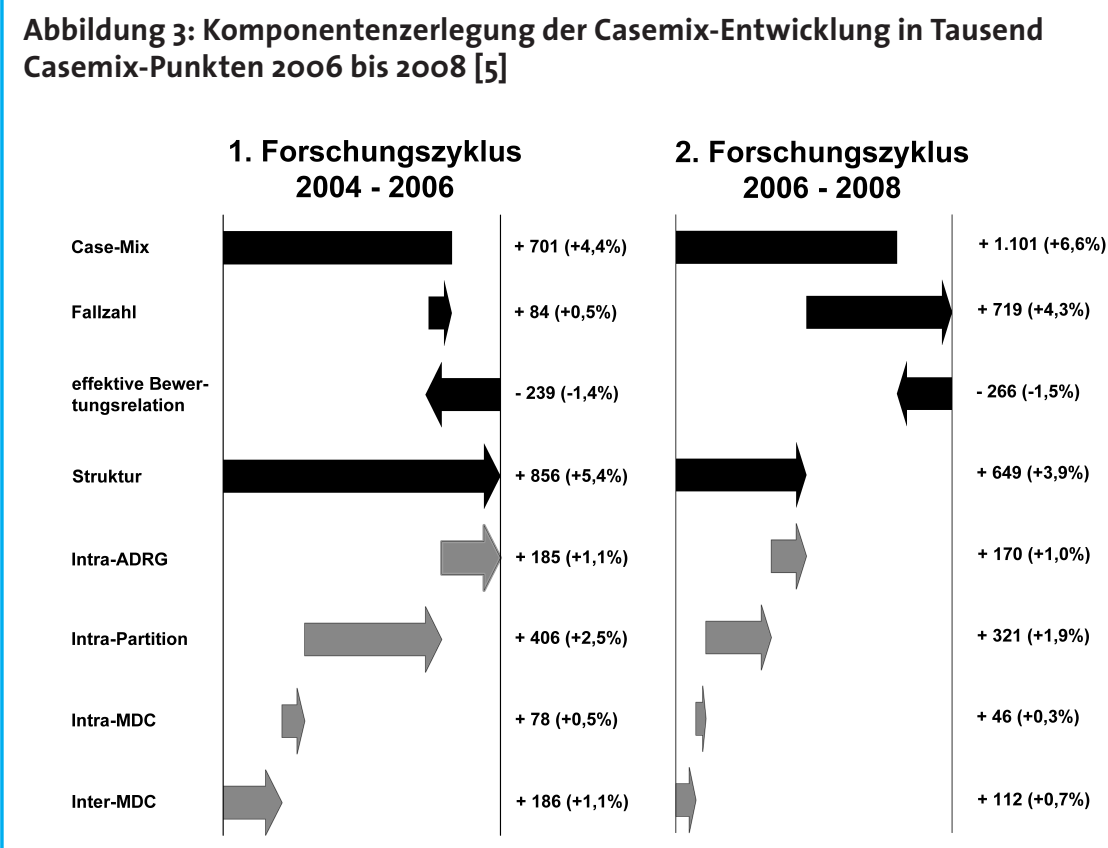

völkerungsrückgangs) allein aufgrund der demografischen Entwicklung eine Zunahme auf rund 19 Mio. Krankenhausfälle jährlich.

Die beschriebene Ausgabenentwicklung muss jedoch nicht notwendigerweise im Anstieg der stationären Behandlungsfälle begründet liegen, sondern kann auch aufgrund morbiditätsbedingter Verschiebungen des Schweregrads von stationären Behandlungsfällen entstehen. In der Regel wird daher auch die Entwicklung des deutschlandweiten Casemix der Daten gemäß $\$ 21$ Krankenhausentgeltgesetz (KHEntgG) ${ }^{6}$ herangezogen, der beide Faktoren berücksichtigt. Tatsächlich lässt sich auf der Basis des Jahres 2004 ein kontinuierlicher Trend zur Casemix-Steigerung verfolgen. Zwischen 2004 und 2010 lag die prozentuale Casemix-Steigerung bei durchschnittlich knapp drei Prozent (sie-
Tabelle 2: Casemix-Änderungen 2004 bis 2010 [4]

\begin{tabular}{|l|c|c|c|}
\hline Jahr & $\begin{array}{c}\text { Casemix gemäß } \\
\$ 21 \text { KHEntgG }\end{array}$ & $\begin{array}{c}\text { Delta Casemix } \\
\text { gegenüber dem } \\
\text { Vorjahr }\end{array}$ & $\begin{array}{c}\text { Prozentuale } \\
\text { Casemix- } \\
\text { Steigerung }\end{array}$ \\
\hline 2004 & 15.476 .804 & - & - \\
\hline 2005 & 16.033 .965 & 557.161 & $3,60 \%$ \\
\hline 2006 & 16.528 .236 & 494.271 & $3,08 \%$ \\
\hline 2007 & 17.148 .731 & 620.495 & $3,75 \%$ \\
\hline 2008 & 17.683 .593 & 534.862 & $3,12 \%$ \\
\hline 2009 & 18.084 .944 & 763.050 & $2,27 \%$ \\
\hline 2010 & 18.559 .432 & 474.488 & $2,62 \%$ \\
\hline
\end{tabular}

Quelle: Statistisches Bundesamt he Tab. 2).

Es stellt sich daher die Frage, welche Faktoren als ursächlich für den Anstieg des deutschlandweiten Casemix zu betrachten sind. Anhand der vom Wissenschaftlichen Institut der AOK (WIdO) entwickelten Methode der Komponentenzerlegung kann empirisch geschätzt werden, welchen Beitrag die unterschiedlichen Faktoren zur Entwicklung des Casemix leisten. Auf diese Weise lässt sich bspw. ermitteln, ob ein höherer Casemix auf Fallzahlsteigerungen oder auf die Abrechnung höher bewerteter DRGs, die einen höheren Schweregrad repräsentieren und dementsprechend mehr Casemix-Punkte einbringen, zurückzuführen ist.

Gliedert man mithilfe der Komponentenzerlegung die beschriebene gesamte Casemix-Steigerung auf, so lassen sich für die zwei durch das IGES Institut bisher betrachteten Drei-Jahres-Zyklen der DRG-Begleitforschung unterschiedliche Entwicklungen identifizieren (siehe Abb. 3).

Der erste Zyklus umfasst die Jahre 2004 bis 2006 und ist von konstanten Fallzahlen (bei steigendem Casemix) gekennzeichnet. Für diese Berichtsperiode dominiert somit der sog. Intrapartitioneneffekt, d. h. die Casemix-Wirkung kam dadurch zustande, dass in der Be-

6 Gesetz über die Entgelte für voll- und teilstationäre Krankenhausleistungen (Krankenhausentgeltgesetz - KHEntgG) vom 23.04.2002, zuletzt geändert durch Art. 7 G v. 22.12.2011 (BGBl. I S. 2983). 
richtsperiode andere Basisfallgruppen innerhalb der gleichen Hauptdiagnosegruppe (Major Diagnostic Category, MDC) und Partition als in der Basisperiode erbracht wurden. Es wurden demnach vermehrt höher bewertete DRGs der gleichen MDC und Partition abgerechnet. Erklären lässt sich dieser Umstand mit den in der Einführungsphase starken Lerneffekten, die eine Steigerung des Casemix zuließen, ohne dass gleichzeitig Fallzahlsteigerungen vorlagen. Zum Nachteil der Krankenkassen ist es in dieser Phase nicht in ausreichendem Maße gelungen, die Ausgabeneffekte der veränderten Kodierung vollständig auszugleichen oder ausgabenneutral zu stellen.

Im Gegensatz zur ersten Berichtsperiode ist der zweite Zyklus der DRG- zent) des zusätzlichen Casemix der Jahre 2006 bis 2008 aus der Prä-MDC sowie aus den operativen Partitionen der MDC-05 und der MDC-08 ergibt (siehe Abb. 4). Dies liegt zum einen an der generellen Bedeutung dieser MDC. Zum anderen zeigen die Analysen auf, dass sich die Fallzahlsteigerung innerhalb dieser drei MDCs nur auf wenige Fallpauschalengruppen konzentriert: DRGs für Defibrillatoren, für die stationäre Wirbelkörperfusion sowie für die totale Knie- und Hüftendoprothetik. Es lassen sich dabei auf der Ebene einzelner DRGs dramatische Fallzahlenausweitungen identifizieren. So stieg bspw. alleine das Casemix-Volumen für Wirbelkörperfusionen zwischen 2006 und 2008 um 20.000 bis 25.000 Casemix-Punkte jährlich. Setzt man einen durchschnitt-

\section{Abbildung 4: Casemix-Veränderung im Zeitraum 2006 bis 2008 nach MDC und Partition [5]}

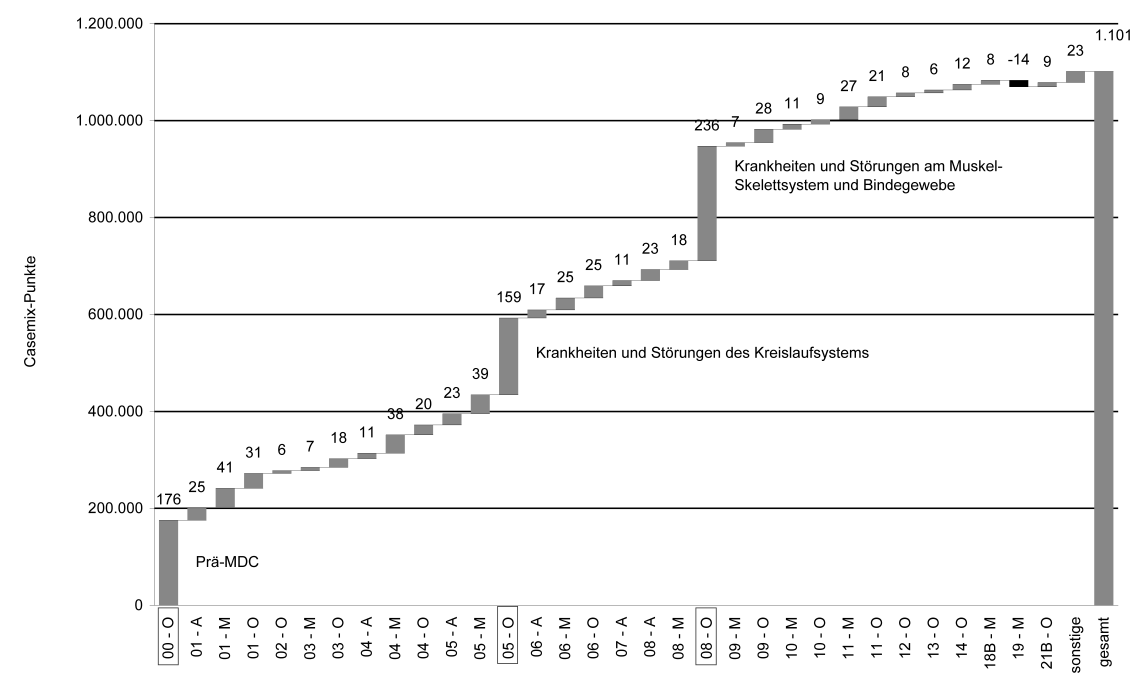

Legende:

MDC oo: Prä-MDC

MDC 05: Krankheiten und Störungen des Kreislaufsystems

MDC 08: Krankheiten und Störungen am Muskel-Skelettsystem und Bindegewebe

Begleitforschung der Jahre 2006 bis 2008 durchgehend von steigenden Fallzahlen gekennzeichnet. So lassen sich 0,7 der 1,1 Mio. Casemix-Punkte auf die Fallzahlentwicklung zurückführen. Zusätzlich lässt sich auch in dieser Berichtsperiode ein substanzieller Intrapartitioneneffekt ausmachen. Dieser ist jedoch insgesamt deutlich schwächer ausgeprägt als zwischen 2004 und 2006.

Eine detailliertere Auswertung auf Ebene der einzelnen MDC zeigt zudem, dass sich mehr als die Hälfte (51 Pro- lichen Basisfallwert von 2.800 Euro an, ergeben sich Zusatzausgaben in Höhe von 56 Mio. Euro nur für diese spezielle Operation (Wolff 2012).

Insgesamt legt die Komponentenzerlegung im Rahmen der DRG-Begleitforschung nahe, dass zwischen 2006 und 2008 der jährliche, altersstandardisierte Casemix-Anstieg in Höhe von 3,3 Prozentpunkten nur in einer Größenordnung von einem Prozentpunkt auf die Alterung der Bevölkerung und damit die erwartete Morbiditätsentwicklung zu-

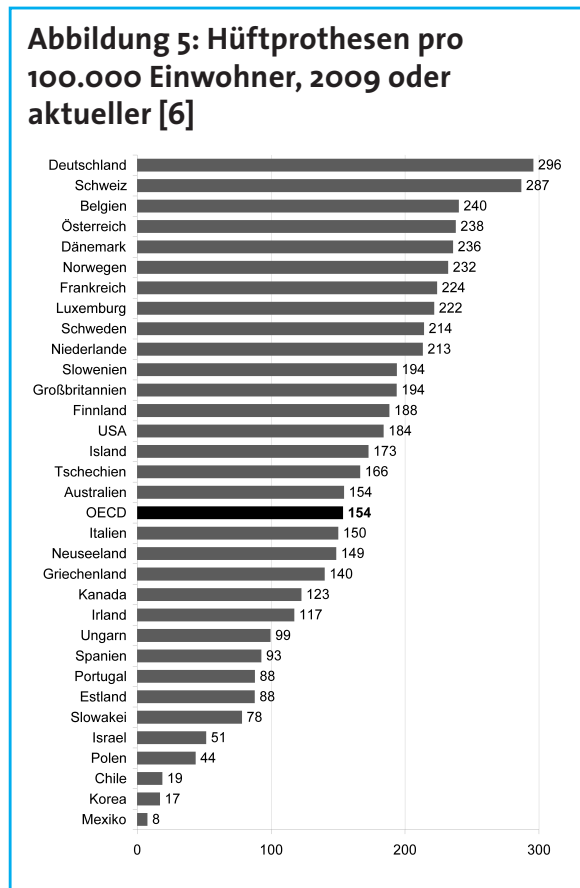

rückzuführen ist (IGES 2011). 2,3 Prozent des Casemix-Anstiegs werden hingegen nicht mit der älter werdenden Bevölkerung und dem daraus steigenden medizinischen Bedarf erklärt. Zu ähnlichen Ergebnissen kommen empirische Analysen des Rheinisch-Westfälischen Instituts für Wirtschaftsforschung (RWI 2012), die weniger als die Hälfte des Casemix-Anstiegs zwischen 2006 und 2010 auf die demografische Entwicklung und eine veränderte Morbiditätsstruktur zurückführen.

Die Leistungsmengenentwicklung und damit auch die Ausgabenentwicklung scheinen sich daher insbesondere für bestimmte Leistungsbereiche von der Morbidität der Bevölkerung zu entkoppeln. Es drängt sich daher der Verdacht auf, dass die GKV nicht nur den Morbiditätsanstieg der Versicherten regelhaft finanziert, sondern auch eine angebotsinduzierte Mengenausweitung.

Insbesondere der Anstieg von Operationen im Bereich der Endoprothetik legt nahe, dass einige der Operationen eher ökonomisch motiviert sind und über das medizinisch Induzierte hinausgehen. Inwieweit dies zutrifft, kann hier nicht beurteilt werden. Auffällig ist allerdings das Ausmaß endoprothetischer Versorgung im internationalen Vergleich, in dem Deutschland die Spitzenposition einnimmt (siehe Abb. 5).

Insgesamt ergibt sich folgendes Bild: Ohne Kapazitätsbegrenzung durch die Krankenhausplanung induzieren die überhöhten Basisfallwerte inzwischen 
eine Mengenentwicklung, die über das medizinisch notwendige Maß hinausgeht. Als Reaktion hat der Gesetzgeber im GKV-FinG eine Mengenbegrenzung über einen Mehrleistungsabschlag von 30 Prozent vorgesehen, der mit dem PsychEntgG für 2013 und 2014 auf 25 Prozent abgesenkt wurde. Jedoch scheinen Mehrleistungsabschläge in dieser Größenordnung kaum geeignet, die Mengenausweitung substanziell zu begrenzen. Denn aus Krankenhausperspektive entscheidet nicht die Höhe des Mehrleistungsabschlags per se darüber, ob eine Mengenausweitung wirtschaftlich attraktiv ist. Vielmehr werden - zumindest in kurzer Frist - die variablen Kosten, d. h. die Kosten, die für die Behandlung eines zusätzlichen Falls einer bestimmten DRG anfallen, im Verhältnis zum (ggf. um den Mehrleistungsabschlag verminderten) Erlös betrachtet. Es entsteht ein Anreiz, die Menge auszuweiten, wenn die variablen Kosten unterhalb des zusätzlichen Erlöses für die neu erbrachte Menge liegen. Untersuchungen des RWI zeigen, dass die durchschnittlichen Grenzkosten aller DRGs ca. 70 Prozent der stationären Behandlungskosten eines Falls ausmachen (RWI 2012). Undifferenzierte Mehrleistungsabschläge von 25 bzw. 30 Prozent, die in gleicher Form für alle DRGs gelten, scheinen daher kein geeignetes Instrument, um angebotsinduzierte Mengenausweitungen zu vermeiden, da der durchschnittliche Erlös die durchschnittlichen variablen Kosten trotz des Abschlags übersteigt.

\section{Forschungsauftrag Mengenentwicklung}

Der Gesetzgeber sieht inzwischen Handlungsbedarf bezüglich der Mengenentwicklung im stationären Bereich. Im Rahmen des PsychEntgG erhalten die Selbstverwaltungspartner auf Bundesebene den Auftrag, einen gemeinsamen Forschungsauftrag mit dem Ziel zu vergeben, insbesondere die Leistungsentwicklung und bestehende Einflussgrößen zu untersuchen sowie gemeinsame Lösungsvorschläge zu erarbeiten und deren Auswirkungen auf die Qualität der Versorgung und die finanziellen Auswirkungen zu bewerten (Neufassung \ 17 b Abs. 9 KHG). Es darf zunächst bezweifelt werden, dass die gemeinsame Vergabe wesentlich zur Qualität der Lö- sungsvorschläge beiträgt.

Problematischer aber noch ist die inhaltliche Vorgabe des Gesetzgebers: „Dabei sind insbesondere Alternativen $\mathrm{zu}$ der Berücksichtigung zusätzlicher Leistungen beim Landesbasisfallwert zu prüfen.“ (\$ 17 b Abs. 9 KHG (neu)). Das kommt einer Aufforderung gleich,

\section{Sinnvoll und kurzfristig umsetzbar sind Mindestmengenregelungen.}

neben der richtigen Lösung (Absenkung der Basisfallwerte) auch möglichst viele falsche Lösungen zu suchen. Wie oben dargestellt ist der Landesbasisfallwert die zentrale Steuerungsgröße. Senkt man den Basisfallwert, dann gibt es auch kein Mengenproblem.

Nochmals: Die derzeitige Mengenproblematik ist durch das Zusammenwirken dreier Effekte entstanden:

- Die Kapazitätsbegrenzung durch die klassische Krankenhausplanung funktioniert nicht mehr.

- Das (gewollt!) leistungsorientierte DRG-System fördert die Erbringung zusätzlicher Leistungen.

- Durch überhöhte Basisfallwerte entsteht ein Anreiz, Leistungen über das medizinisch Sinnvolle hinaus zu erbringen.

Da weder die klassische Krankenhausplanung wieder restauriert werden kann und es außerdem sinnvoll ist, das DRG-System zu erhalten, läge es eigentlich nahe, eine regelbasierte Anpassung der Landesbasisfallwerte vorzunehmen. Dass diese jedoch das Ergebnis einer gemeinsamen Lösungssuche von DKG und GKV-Spitzenverband sein wird, ist ziemlich unwahrscheinlich.

Kleine Nebenbemerkung: Einem allgemeinen Trend folgend enthält der Forschungsauftrag gemäß $\$ 17$ b Abs. 9 KHG (neu) auch das Wort „Qualität“. Es gilt Möglichkeiten zur Stärkung qualitätsorientierter Komponenten in der Leistungssteuerung zu entwickeln. Die Erfahrungen bei der sektorübergreifenden Qualitätssicherung, in der selbst sieben Jahre nach der gesetzlichen Beauftragung kein einziges Verfahren gestartet sein wird, zeigen, dass eher mit einer längerfristigen Entwicklung zu rechnen ist. Sinnvoll und kurzfristig umsetzbar sind allerdings Mindestmengenregelungen; es bedürfte lediglich einer Beseitigung gesetzlicher Hürden zum Beschluss solcher Regelungen. Mittelfristig ist vor allem an eine Innovationsbewertung zu denken. Ein Teil der Mengenproblematik ist auch Folge vermeintlich innovativer Leistungen ohne Nutzennachweis.

Hier heißt es, den $\mathbb{} 137$ e SGB V (neu) strenger zu fassen.

Kern des Forschungsauftrages dürften jedoch die im engeren Sinne ökonomischen Steuerungsoptionen sein. Einige zentrale Ansätze seien im Folgenden kurz skizziert: regelbasierte Anpassung der Landesbasisfallwerte, Ausschreibung elektiver Leistungen, krankenhausspezifische Mehrleistungsabschläge und Transfer von elektivem Casemix.

\subsection{Regelbasierte Anpassung der Landesbasisfallwerte}

Wie beschrieben ist seit der Einführung des DRG-Systems die Anpassung der Landesbasisfallwerte quasi jährlich Gegenstand gesetzgeberischer Aktivitäten. Formal handelt es sich um einen Verhandlungsprozess zwischen Kassen und Krankenhäusern auf Landesebene, aber die Vorgaben für diesen Verhandlungsprozess (Obergrenzen, Berücksichtigung von Tarifsteigerungen etc.) gleichen fast in keinem Jahr denen des Vorjahres. Eine traditionelle Begrenzung für Preissteigerungen stellte ursprünglich die Grundlohnorientierung dar, deren Wirkung jedoch häufig falsch interpretiert wird. Insbesondere vor dem Hintergrund der Einführung des Kostenorientierungswertes, der die Grenze für Preissteigerungen weiter aufweicht, muss daher klar darauf hingewiesen werden, dass die öffentliche Diskussion oftmals an zwei fundamentalen Irrtümern krankt.

Der erste Irrtum ist die Verwechslung von Budget- und Preisregulierung. Die Obergrenze für Steigerungen der Landesbasisfallwerte wird wie die Begrenzung einer Budgetsteigerung diskutiert. Das ist sie aber mitnichten. Landesbasisfallwerte sind Preise. Da in den letzten Jahren kontinuierliche Mengensteigerungen in Höhe von fast drei Prozent zu verzeichnen sind, ergibt sich jährlich eine Ausgaben- bzw. Budgetsteigerung, die deutlich über die Obergrenze hinausgeht. Eine Grundlohnorientierung für die Landesbasisfallwerte bedeutet eben 


\section{Abbildung 6: Indexierte Kosten der Krankenhäuser und indexierte Kosten der Krankenhäuser je Casemix-Punkt 2005 bis 2010 [7]}

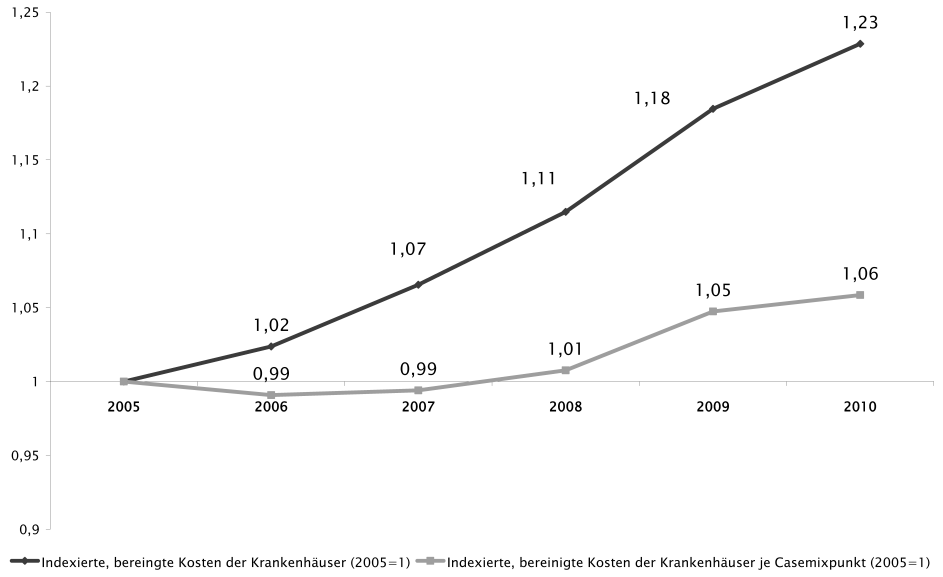

nicht, dass die Budgets der Krankenhäuser auf die Grundlohnentwicklung begrenzt werden. Und eine Grundlohnorientierung bei Landesbasisfallwerten garantiert auch keinerlei Beitragssatzstabilität. Die Grundlohnorientierung als Preisobergrenze ist viel zu hoch!

Der zweite Irrtum betrifft die Kostenentwicklung. Gemäß der herrschenden sozialpolitischen Logik des Orientierungswertes sollen „externe“ Kostensteigerungen die gesundheitliche Versorgung nicht gefährden. So soll bspw. eine Ölpreissteigerung nicht die Krankenhäuser in Bedrängnis bringen, sondern via Basisfallwerterhöhung an die Beitragszahler weitergegeben werden. Folgt man dieser Logik, so muss gleichwohl kritisch gefragt werden, ob denn die Krankenhäuser aufgrund externer Kostenentwicklung wirklich in Schwierigkeiten geraten oder ob es nicht kompensatorische Effekte gibt, die die Entwicklungen auf der Kostenseite ausgleichen. So kann z. B. der medizinisch-technische Fortschritt eine Verweildauerverkürzung ermöglichen, die dafür sorgt, dass mit demselben „Input“ mehr DRG-Fälle abgerechnet werden können.

Vereinfacht sei dies an der Blinddarmoperation illustriert. Der „Gesundheitspolitiker“ will, dass die (notwendige) Blinddarmoperation unabhängig von der Wirtschaftsentwicklung finanziert werden soll. Kostensteigerungen werden somit implizit akzeptiert. Auch ein gröBerer Bedarf an Blinddarmoperationen (Morbi-Entwicklung) sollte zulasten der Kassen gehen. Daraus folgt, dass sich die Preisentwicklung an der Kostenentwick- lung je Blinddarmoperation - also den Fallkosten (genauer: Kosten je CasemixPunkt) - orientieren sollte.

Eine sinnvolle, regelbasierte Fortschreibung der Landesbasisfallwerte würde also lediglich jene Kostensteigerung wirksam werden lassen, die aufgrund fehlender kompensatorischer Erlöseffekte die Krankenhäuser wirklich in Schwierigkeit bringt. Es sollte also die Kostenentwicklung je Casemix-Punkt vom Statistischen Bundesamt gemessen und als Basis für den Orientierungswert

\section{Die Kostensteigerungen quasi amtlich durch das Statistische Bundesamt ermitteln zu lassen, muss zu überhöhten Landesbasisfallwerten führen.}

zugrunde gelegt werden. Statt einer rohen Kostensteigerung von 23 Prozent in den Jahren 2005 bis 2010 ergibt sich dann eine Größenordnung von sechs Prozent (siehe Abb. 6).

Gegen eine Berücksichtigung der Produktivitätsentwicklung bei einer regelbasierten Anpassung wird bisweilen eingewendet, dass diese Gegenstand auf der Landesebene ist. Dies ist in der Tat der Fall, gilt aber in gleichem Maße für die Kostenentwicklung. Ordnungspolitisch ergeben sich zwei stimmige Lösungsoptionen:

nosten- und Produktivitätsentwicklung werden ausschließlich auf der Landesebene ermittelt und verhandelt.
- Sowohl Kosten- als auch Produktivitätsentwicklung werden auf Bundesebene ermittelt und verhandelt.

Die gegenwärtige Lösung, die Kostensteigerungen quasi amtlich auf Bundesebene durch das Statistische Bundesamt ermitteln zu lassen, die Produktivitätsentwicklung aber einem vagen, in der Regel wenig regelbasierten Verhandlungsprozess zu überlassen, muss $\mathrm{zu}$ überhöhten Landesbasisfallwerten führen.

\subsection{Ausschreibung elektiver Leistungen}

Ausschreibungen setzen ebenfalls an der Preisseite an und sehen vor, dass der beidseitige Kontrahierungszwang zwischen Krankenkassen und Krankenhäusern für Teile des Leistungsspektrums entfällt. Dabei bleibt die Notfallversorgung weiterhin Gegenstand planerischer Entscheidungsprozesse auf Landesebene, während sich die Kompetenz der Bundesländer für planbare Leistungen auf die Festlegung von Mindestvorgaben (Leistungsmengen) und die Kontrolle zu deren Einhaltung beschränkt. Krankenkassen und Krankenhäuser verhandeln dann im Rahmen von Selektivverträgen die stationären Mengen und den Preis von elektiven Leistungen. Für eine Harmonisierung unter den Bundesländern sollte der Gemeinsame Bundesausschuss (GBA) bundesweite Versorgungsrichtgrößen vorgeben. Die Krankenkassen sichern in diesem Rahmen die Vorgaben durch Ausschreibung ab.

Detaillierte Ausarbeitungen zu den notwendigen Rahmenbedingungen, um eine bedarfsgerechte Versorgung sicherzustellen, liegen vor (Leber et al. 2007).

Mit Selektiverträgen kann die Mengendynamik vieler stationärer Krankenhausleistungen wirkungsvoll gesteuert werden. Bei entsprechender Ausgestaltung der Rahmenbedingungen sind keine negativen Auswirkungen auf die Versorgungsqualität oder eine Gefährdung der Versorgungssicherheit zu befürchten. Allerdings führen Direktverhandlungen zwischen Krankenkassen und Krankenhäusern zu einer Erhöhung der Transaktionskosten. Zudem sind strukturelle Reformen notwendig, damit selektivvertragliche Mechanismen ihre Steuerungswirkung entfalten können. 


\subsection{Krankenhausspezifische Mehrleistun gsabschläge}

Eine Kombination von Mengen und Preisansatz stellen krankenhausspezifische Mehrleistungsabschläge dar, die schon jetzt, wie beschrieben, im KHG verankert sind. Der Abschlag wird momentan als einheitlicher Abschlag vom Landesbasisfallwert umgesetzt. Allerdings muss die derzeitige gesetzliche Regelung angepasst werden, um der Mengendynamik entgegenzuwirken, denn nur variable Kosten fallen bei zusätzlicher Erbringung der Leistungen im Krankenhaus erneut an. Krankenhausspezifische Mehrleistungsabschläge müssen daher deutlich höher ausfallen als derzeit vom Gesetzgeber vorgesehen, wenn sie ökonomisch motivierte Mengenausweitungen verhindern sollen. Zielführend scheinen, ausgehend von vorliegenden Zahlen des RWI (RWI 2012) sowie aus der InEK-Kalkulation, Mehrleistungsabschläge zwischen 65 und 100 Prozent. Zudem dürfen Mehrleistungsabschläge nicht erhöhend bei den Verhandlungen zum Landesbasisfallwert berücksichtigt werden, wenn weiterhin nach $\mathbb{1 0}$ Abs. 1 Satz 2 KHEntgG ausgeschlossen wird, dass die gekürzten Krankenhausbudgets im Folgejahr den Landesbasisfallwert absenken. Ansonsten wird die mengen- und ausgabenabsenkende Wirkung des laufenden Jahres im Folgejahr wieder aufgehoben und das Casemix-Volumen für die Krankenkassen im Folgejahr steigt über das Niveau vor der Einführung eines Mehrleistungsabschlags. Bei konsequenter Umsetzung krankenhausspezifischer Mehrleistungsabschläge kann durch eine degressive Vergütung eine ökonomisch motivierte Mengenausweitung eingegrenzt werden.

Alternativ könnten ausgehend von der InEK-Kalkulation DRG-spezifische degressive Vergütungsalgorithmen (RWI 2012) entwickelt werden. Dieser Ansatz setzt nicht am Landesbasisfallwert an, sondern staffelt Relativgewichte nach Fallzahlen. Konkret bedeutet dies, dass Krankenhäuser je nach Fallzahl unterschiedliche Relativgewichte und Fallpauschalen realisieren. Beide Formen der degressiven Vergütung können im momentanen kollektivvertraglichen Rahmen umgesetzt werden und gehen mit begrenzten Transaktionskosten einher.
Bei der Beurteilung solcher krankenhausspezifischer Steuerungsmechanismen sollte beachtet werden, dass sie zwar kurzfristig wirksam sind, dass sie aber erheblichen ordnungspolitischen Schaden anrichten, weil sie letztlich immer auf eine strukturelle Erstarrung hinauslaufen. Sie bedeuten auch eine Abkehr vom Ziel einer leistungs- und qualitätsorientierten Vergütung. Würden z. B. im Falle erheblicher Qualitätsveränderungen große Teile des Leistungsvolumens von einem schlechteren zu einem besseren Krankenhaus wandern, dann sollte in einem leistungs- und qualitätsorientierten System auch das Finanzierungsvolumen wandern („Geld folgt Leistung“). Krankenhausspezifische Abschläge würden verhindern, dass die Leistungsausweitung im besseren Haus adäquat finanziert wird.

Ähnliche Vorbehalte müssen gegen

Kann eine effizientere Versorgung erreicht werden, wenn Abrechnungslizenzen übertragbar und gewissem Sinne handelbar gemacht werden können?

fallzahlabhängige Vergütungen gemacht werden. Sie verhindern, dass sich eine aus Qualitäts- und Produktivitätsgründen sinnvolle Spezialisierung entwickelt. Man denke an den Fall zweier benachbarter Krankenhäuser mit Doppelvorhaltung von Fachabteilungen, bei denen eine strukturelle Bereinigung durch Konzentration auf jeweils ein Fachgebiet sinnvoll wäre. Bei fallzahlabhängiger Vergütung wäre dies - unsinnigerweise - mit Vergütungsabschlägen verbunden. Grundsätzlich sollte eher nach Lösungen gesucht werden, die eine strukturelle Weiterentwicklung der Krankenhauslandschaft ermöglichen. Der folgende Vorschlag stellt eine solche Überwindung erstarrter Strukturen in den Mittelpunkt.

\subsection{Lizenzierung und Transfer von elektivem Casemix}

Ausgangspunkt für die seit Kurzem diskutierten Konzepte zum Transfer von Leistungsmengen ist der Tatbestand, dass die Aufnahme in den Krankenhausplan eine Abrechnungslizenz dar- stellt. Durch Kontrahierungszwang sind Kassen und Versicherungen gezwungen, Leistungen von Plankrankenhäusern zu finanzieren. Es stellt sich die Frage, ob eine effizientere Versorgung erreicht werden kann, wenn diese Lizenzen übertragbar und in gewissem Sinne handelbar gemacht werden können. Dieses Konzept orientiert sich an Ansätzen, die u. a. in der Umweltpolitik genutzt werden, wo Zertifikate handelbar gemacht worden sind, um einen vom Staat vorgegebenen oder kollektivvertraglich vereinbarten Standard zu erreichen und dabei minimale volkswirtschaftliche Kosten zu generieren.

Für den Gesundheitsbereich mag eine solche Betrachtung zunächst ungewohnt erscheinen, de facto ist aber der Kauf von Abrechnungslizenzen seit Langem fester Bestandteil der Versorgungssteuerung. So ist bspw. der Kauf und Verkauf von Kassenarztsitzen nichts anderes als Erwerb bzw. Veräußerung einer Abrechnungslizenz gegenüber der gesetzlichen Krankenversicherung. Auch der Verkauf eines Plankrankenhauses durch den Träger stellt nichts anderes dar als die Veräußerung einer Abrechnungslizenz. Der Eintritt privater Krankenhausträger auf den Krankenhausmarkt funktioniert in Deutschland quasi ausschließlich über den Erwerb von bestehenden Abrechnungslizenzen, also den Aufkauf von Plankrankenhäusern. In all jenen Fällen, bei denen veraltete Krankenhäuser durch komplett eigenfinanzierte Neubauten ersetzt wurden, wird besonders deutlich, dass der Kaufpreis letztlich nicht für die bestehenden Gebäude, sondern für eine Lizenz bezahlt wird. Die Listung in einem Krankenhausplan ist ein millionenschwerer Wert.

Es stellt sich die Frage, inwieweit nicht nur ganze Krankenhäuser, sondern auch Teile der Abrechnungslizenz veräußerbar gemacht werden können. Und es stellt sich die Frage, ob die Standortbindung der Lizenz in gewissem Grade aufgehoben werden kann. Dann wären Lizenzen auch an andere Krankenhäuser transferierbar.

Ähnlich wie beim direktvertraglichen Ansatz müssen Notfallleistungen und planbare (elektive) Leistungen getrennt betrachtet werden. Die Notfallversorgung bleibt weiterhin Gegenstand detaillierter planerischer Entscheidungs- 
prozesse. Elektive Leistungen hingegen könnten unter Einhaltung bestimmter Regularien transferiert werden. Im Folgenden sei vom Transfer des elektiven Casemix (eCM) gesprochen.

Mit einer eCM-Lizenz erhält jedes Krankenhaus nicht nur die Erlaubnis, bestimmte elektive Leistungen zu einem weiterhin kollektivvertraglich festgesetzten Preis zu erbringen, sondern kann diese alternativ an andere Krankenhäuser transferieren, die zusätzliche Mengen erbringen wollen. Für den Transfer von eCM-Teillizenzen erhält das abgebende Krankenhaus vom Empfänger der Lizenz eine finanzielle Kompensation, die bspw. zur Krankenhausentwicklung eingesetzt werden kann.

Eine Lösung des Mengenproblems ergibt sich dann, wenn das eCM-Volumen quasi auf Makroebene festgeschrieben wird. Ausgangspunkt wäre z. B. das eCM-Volumen eines Bundeslandes im Status quo. Schreibt man dieses fest oder schreibt es nach bestimmten Regeln fort, dann ist eine Mengenausweitung im einzelnen Krankenhaus nur noch durch Zukauf von Lizenzen möglich. De facto entspricht das einem hundertprozentigen Mehrleistungsabschlag, der aber durch Erwerb von Casemix anderer Häuser „entschärft" würde. Ohne eine solche krankenhausspezifische Mehrleistungsbeschränkung wäre das Konzept schlichtweg die Auflösung jeder Planung mit völlig ungesteuerter Leistungsvermehrung.

Die Lizenzierung von eCM ist geeignet, die Leistungserbringung von ökonomisch motiviert induzierten Mengen einzuschränken. Gleichzeitig bleiben Anreize für eine effizientere Leistungserbringung durch Spezialisierung erhalten, denn Krankenhäuser mit niedrigen Produktionskosten in bestimmten Leistungsbereichen können durch den Transfer von eCM-Lizenzen Mengen ausweiten. Hingegen werden notwendige Neu- oder Umstrukturierungen aufgrund zu hoher Produktionskosten (z. B. das Schließen einer Abteilung) für die betroffenen Krankenhäuser abgefedert, da sie für den Transfer der entsprechenden eCM-Lizenzen eine finanzielle Kompensation erhalten. Durch die Berücksichtigung des Opportunitätskostenprinzips, d. h. Krankenhäuser entscheiden individuell, wie sie mit ihren und den als Transfer verfügbaren eCM-Lizenzen verfahren, wird zudem die starre Ineffizienz von direkten regulativen mengenseitigen Eingriffen vermieden. Auch die Trial-and-Error-Problematik von krankenhausindividuellen Mehrleistungsabschlägen sowie die hohen Transaktionskosten von direktvertraglichen Lösungen werden umgangen.

Ein wichtiger Vorteil dieses Ansatzes ist zudem, dass auf der Preisseite der Rahmen des kollektivvertraglich eingebetteten G-DRG-Systems nicht verlassen werden muss. Ähnliches gilt für die Qualitätssicherung: Alle Instrumente, Mechanismen und Anforderungen an die Struktur-, Prozess- und Ergebnisqualität können im Rahmen des Lizenzierungsansatzes im gleichen Maße wie im Status quo angewendet werden. Sie gehen mit ähnlichen Stärken und (insbesondere) Schwächen einher. Allerdings führt der Lizenzierungsansatz von eCM zu einem erhöhten Reform- und Bürokratieaufwand, da eine Institution geschaffen werden muss, die den Transfer von eCM-Lizenzen abwickelt, dokumentiert und überwacht.

Die Regularien, unter denen ein Transfer möglich ist, bedürfen intensiver Diskussion. So ist insbesondere zu regeln, ob der Casemix (abteilungsbezogen) spezifiziert werden sollte, ob der Casemix beliebig weit handelbar sein sollte, ob Investitionen der Länder den Transfer einschränken und ob Einspruchsrechte der „klassischen Akteure" (Planungsbehörden, Krankenkassen, Kartellamt) kodifiziert werden sollten. Dazu einige kurze Anmerkungen.

\section{Casemix-Spezifikation}

Ein unspezifizierter Casemix würde zur Abrechnung beliebiger DRG-Leistungen berechtigen. Dadurch könnte der Casemix einer orthopädischen Abteilung nach Transfer zur Abrechnung geriatrischer Leistungen berechtigen und umgekehrt. Das mag auf den ersten Blick bedenklich erscheinen, ist aber schon heute Realität. Im Rahmen des geltenden Krankenhausrechts besteht bezüglich des Casemix weitestgehend Anbieterautonomie. Letztlich entscheidet das Krankenhaus, welche Leistungen es erbringt. Vermehrt ein Krankenhaus seine chirurgischen Leistungen auf Kosten anderer Abteilungen, so ist dies letztlich von Planungsbehörden und Kostenträgern zu akzeptieren. Die gerichtlich durchsetzbaren Mitwirkungsrechte der Kassen bei sog. Strukturgesprächen sind ausgesprochen bescheiden. Das Budgetrecht macht letztlich keinen Unterschied zwischen vereinbarten und nicht vereinbarten DRG-Volumina. Das Konzept der „Rahmenplanung“ erhebt diese Anbieterautonomie sogar zur Doktrin. Der Unterschied zur jetzigen Situation besteht also allein darin, dass die Stand- 
Bundeskartellamtes sein. Gleichermaßen notwendig dürften Einspruchsrechte von Landesplanungsbehörden und Krankenkassen sein, um den Transfer aus unterversorgten in überversorgte Gebiete zu unterbinden. Dies verlangt allerdings die Definition von (bundeseinheitlichen) kriteriengebundenen Einspruchsrechten, da es andernfalls zum kompletten Stillstand jeglicher Transfers kommen würde.

Der große Vorteil eines Konzepts zum eCM-Transfer besteht letztlich in der Standortbereinigung in Ballungsgebieten. Diese ist unter den Bedingungen des herrschenden Krankenhausrechts nicht gelungen. Es existieren weiterhin zahlreiche kleine Krankenhäuser mit ineffizienten „Produktionsmengen“ in Fußgängerentfernung zum nächsten kleinen Krankenhaus - mit negativen Folgen für die Effizienz und vor allem

\section{Eine rationale, regelgebundene Fortschreibung der Landesbasisfallwerte unter Berücksichtigung von Kosten- und Produktivitätsentwicklung bleibt die zentrale Aufgabe.}

mit negativen Folgen für die medizinische Qualität. Im gegenwärtigen Krankenhausrecht bedeutet die Schließung von Standorten eine Art von entschädigungsloser Enteignung. Bei Veräußerung der Lizenz in einem Konzept mit handelbarem Casemix könnte bspw. ein kirchlicher Träger eine weniger verlustreiche Umstrukturierung von Krankenhausleistungen auf Leistungen der Altenpflege realisieren.

Expandierende Krankenhausträger und Krankenhausträger, die sich verlustfrei anderen Aktivitäten zuwenden wollen, dürften dann auch die wesentlichen Promotoren eines solchen Konzeptes sein. Zurückhaltung dürfte es bei jenen geben, die bislang planerisch und mitgestaltend tätig waren - die Landesbehörden, aber auch die gesetzlichen Krankenkassen.

\section{Vom Forschungsauftrag zur Reform: Ein Ausblick}

Der $\int 17$ b ist im KHG der Ort für Projektskizzen. 1999 wurde hier in wenigen
Sätzen das DRG-System auf den Weg gebracht. Wenn nun wiederum im $\ 17 \mathrm{~b}$ ein Forschungsauftrag zur Steuerung der Krankenhausleistungen verortet wird, dann darf das als Beginn eines längeren Prozesses zur Neuordnung der Krankenhausleistungen gewertet werden. Der Ausgangspunkt ist das Versagen der klassischen Kapazitätssteuerung durch länderfinanzierte Vorhaltung von Krankenhausbetten. Dieses Modell der dualen Finanzierung ist am Ende. Die Krankenhausinvestitionen werden nur noch zum Teil durch die Länder finanziert. Die Krankenhausplanung steuert die Kapazitäten nicht mehr und beschränkt sich weitestgehend auf eine Festlegung von Standorten. Eine nennenswerte Standortbereinigung hat bislang nicht stattgefunden. Da in den Bundesländern positive Versorgungsund Arbeitsmarkteffekte eintreten, aber Folgekosten bundesweit umgelegt werden, war und ist mit einer solchen Bereinigung auch nicht zu rechnen. Standorte im Krankenhausplan sind aufgrund des Kontrahierungszwangs Abrechnungslizenzen gegenüber den Krankenkassen. Struktur und Umfang der stationären Leistungen werden im Wesentlichen autonom von den Anbietern bestimmt. Die wesentliche gesundheitspolitische Steuerungsgröße für Krankenhausleistungen ist seit Einführung der DRG-Fallpauschalen der (Landes-)Basisfallwert. Die bisherigen gesetzlichen Vorgaben zur Fortschreibung der Landesbasisfallwerte änderten sich quasi jährlich und waren im Ergebnis zu hoch. Die dreifache Dynamik von Überkapazitäten, überhöhten Preisen und den Anreizwirkungen des leistungsorientierten DRG-Systems hat zu einer Leistungs- und Ausgabendynamik geführt, die der Gesetzgeber inzwischen als korrekturbedürftig ansieht. Aus Sicht der GKV bleibt eine rationale, regelgebundene Fortschreibung der Landesbasisfallwerte unter Berücksichtigung von Kosten- und Produktivitätsentwicklung die zentrale Aufgabe. Ergänzend sind für elektive, standardisierte Massenleistungen Direktverträge vorzusehen. Sofern es aufgrund von krankenhausbezogenen Begrenzungsmechanismen zur Gefahr struktureller Erstarrung kommen sollte, sind auch Modelle zum Transfer von eCM denkbar. Das traditionelle Kran- kenhausplanungsinstrumentarium würde nur noch im Bereich der Notfallversorgung Anwendung finden.

\section{Quellen:}

[1] DKG, Bestandsaufnahme zur Krankenhausplanung und Investitionsfinanzierung in den Bundesländern, Stand: September 2010, im Internet abrufbar unter www.dkgev.de

[2] führen und wirtschaften im Krankenhaus, Der Flickenteppich kommt (f\&w 2012)

[3] Amtliche Statistik KJ1 (2000 - 2010), KV45 (2011), Schätzungen des GKV-Spitzenverbandes (2012); Darstellung des GKVSpitzenverbandes

[4] Katalogpräsentationen des InEK der Jahre 2005 bis 2011, Daten gemäß $\$ 21 \mathrm{KHEntgG}$ [5] InEK, G-DRG-Begleitforschung gemäß \$17b Abs. 8 KHG (IGES-Institut)

[6] OECD Health Data 2011

[7] Destatis Fachserie 12 Reihe 6.3 (Statistisches Bundesamt 2012) und Daten nach \$21 KHEntgG

\section{Literatur}

führen und wirtschaften im Krankenhaus (f\&w 2012): Der Flickenteppich kommt, 29, 3: 268-269.

IGES (2011): G-DRG-Begleitforschung gemäß $\$ 17$ b Abs. 8 KHG. Endbericht des zweiten Forschungszyklus (2006 bis 2008): Untersuchung im Auftrag des deutschen DRG-Instituts (InEK). Berlin. http://www.g-drg.de/cms/ content/view/full/2944.

Leber WD, Wolff J (2012): Wer bestellt, muss bezahlen. führen und wirtschaften im Krankenhaus (f\&w), 3: 258-261.

Leber WD, Malzahn J, Wolff J (2007): Elektiv wird selektiv. In: Klauber J, Robra BP, Schellschmidt H (Hrsg.): Krankenhaus-Report 2007 Schwerpunkt: Krankenhausvergütung - Ende der Konvergenzphase? Stuttgart: Schattauer, 81-106.

OECD (2011): Health at a Glance 2011: OECD Indicators, OECD Publishing. http://dx.doi org/10.1787/health_glance-2011-en.

Olson M (1969): The Principle of „Fiscal Equivalence“: The Division of Responsibilities among Different Levels of Government, The American Economic Review, 59, 2:479-487.

Rheinisch-Westfälisches Institut für Wirtschaftsforschung (RWI 2012) Mengenentwicklung und Mengensteuerung stationärer Leistungen - Endbericht: Forschungsprojekt im Auftrag des GKV-Spitzenverbands, Essen: RWI.

Statistisches Bundesamt (2012): Destatis Fachserie 12 Reihe 6.3-2010. Gesundheitswesen. Kostennachweis der Krankenhäuser. Wiesbaden. https://www.destatis.de/DE/ Publikationen/Thematisch/Gesundheit/ Krankenhaeuser/GrunddatenKrankenhaeuser html.

Wolff J (2012): Der Preis macht die Menge. Führen und Wirtschaften im Krankenhaus $(f \& w)$, 2: 138-142. 